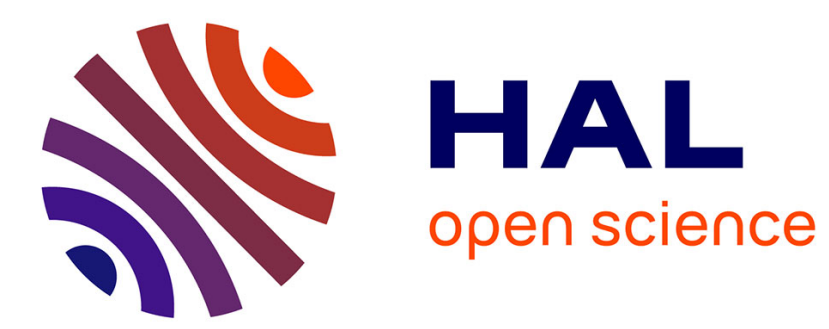

\title{
A generalization of the expenditure function
}

Noé Biheng

\section{- To cite this version:}

Noé Biheng. A generalization of the expenditure function. 2014. halshs-01143222

\section{HAL Id: halshs-01143222 \\ https://shs.hal.science/halshs-01143222}

Submitted on 17 Apr 2015

HAL is a multi-disciplinary open access archive for the deposit and dissemination of scientific research documents, whether they are published or not. The documents may come from teaching and research institutions in France or abroad, or from public or private research centers.
L'archive ouverte pluridisciplinaire HAL, est destinée au dépôt et à la diffusion de documents scientifiques de niveau recherche, publiés ou non, émanant des établissements d'enseignement et de recherche français ou étrangers, des laboratoires publics ou privés. 


\section{Documents de Travail du Centre d'Economie de la Sorbonne}

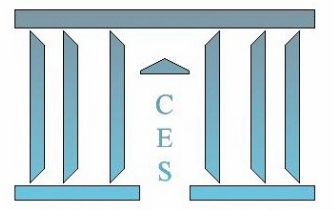

A generalization of the expenditure function

Noé BIHENG

2014.83

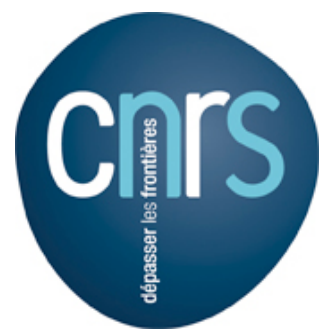

Maison des Sciences Économiques, 106-112 boulevard de L'Hôpital, 75647 Paris Cedex 13 


\title{
A generalization of the expenditure function*
}

\author{
Noé Biheng ${ }^{\dagger}$
}

November 30, 2014

\begin{abstract}
We consider a generalized expenditure function and the associated Hicksian demand. First, we provide some economic interpretation of the problem that we study. Then, we study different properties of the solution: existence, Lipschitz behavior and differential properties. We conclude by a Slutsky-type property.
\end{abstract}

Keywords: expenditure function, incomplete preferences, Lipschitz behavior.

JEL Classification: C6, D4.

\section{Introduction}

Let $u_{1}, \ldots, u_{n}$ be $n$ functions defined on $\mathbb{R}_{++}^{\ell}$.

We study the following optimization problem :

$$
\left\{\begin{array}{l}
\max -p \cdot x \\
\text { subject to } u_{k}(x) \geq v_{k}, k=1, \ldots, n \\
x \gg 0
\end{array}\right.
$$

with $p$ belonging to $\mathbb{R}_{++}^{\ell}$ and $v:=\left(v_{k}\right)_{k=1}^{n} \in \mathbb{R}^{n}$. The solution of this problem will be denoted by $\Delta(p, v)$ and called the generalized Hicksian/compensated demand. The aim of the paper is to study the properties of this mapping. Since this paper is motivated by some economic considerations, we first present five applications.

*I would like to thank Jean-Marc Bonnisseau for his support and Alain Chateauneuf for his valuable comments. All remaining errors are mine.

${ }^{\dagger}$ Paris School of Economics, Université Paris 1 Panthéon Sorbonne, 106-112 boulevard de l'Hôpital 75647 Paris Cedex 13, Noe.Biheng@univ-paris 1.fr 


\subsection{Generalization of the classical compensated demand}

If $n$ is equal to one, $\Delta(p, v)$ is the so-called compensated demand or Hicksian demand $^{1}$. So $\Delta(p, v)$ can be viewed as a multi-criterion extension of the Hicksian demand.

\subsection{Compensated function of the minimum}

Let us consider a consumer whose utility function has the form: $U(x):=\min _{1 \leq k \leq n} u_{k}(x)$. The compensated demand and the expenditure function associated to $U$ are respectively the solution and the value function of Problem 1.1 when we take $v:=\left(v_{k}\right)_{k=1}^{n}=(\bar{v}, \ldots, \bar{v})$.

\subsection{Incomplete preferences}

Let us consider a consumer with "Bewley-type" preferences ${ }^{2}$. The noncomplete preference relation $\succeq$ is defined by: for $(x, y) \in\left(\mathbb{R}_{++}^{\ell}\right)^{2}, x \succeq y$ whenever $u_{k}(x) \geq u_{k}(y)$ for every $k \in\{1, \ldots, n\}$. Let us consider now an element $\bar{x} \in \mathbb{R}_{++}^{\ell}$ and $\left(v_{k}\right)_{k=1}^{n}:=\left(u_{k}(\bar{x})\right)_{k=1}^{n}$. The preferred set of $\bar{x}$ is: $\operatorname{Pref}(\bar{x}):=\left\{x \in \mathbb{R}_{++}^{\ell} \mid u_{k}(x) \geq u_{k}(\bar{x}), 1 \leq k \leq n\right\}$. Consequently, $\Delta(p, v)$ is the compensated demand associated to $\succeq$. A legitimate question would be to ask if there is any duality ${ }^{3}$ between the demand associated with this preference relation and $\Delta(p, v)$. In fact, there is none as it can be easily seen. Indeed, the demand tend to be multi-valued even while the compensated demand is unique when defined.

\subsection{Public goods}

The following application concerns economic planning. Consider an economy with $n$ consumers, $\ell$ public goods ${ }^{4}$ and one private good. Suppose that the level of private good $\xi_{k}$ to be consumed by consumer $k$ has already been chosen, i.e. $u_{k}(x):=U_{k}\left(x, \xi_{k}\right)$ where $U_{k}$ is the utility function of consumer $k$. An economic planner who wants to choose the cheapest basket of public goods with respect to the price $p \in \mathbb{R}_{++}^{\ell}$ given the individual levels of private $\operatorname{good}\left(\xi_{k}\right)_{k=1}^{n}$ has to solve Problem 1.1.

\footnotetext{
${ }^{1} \mathrm{~A}$ presentation of the expenditure minimization problem can be found in any intermediary or advanced microeconomics textbook. For the sake of completeness, we refer the reader to [14].

${ }^{2}$ We refer the reader to [5].

${ }^{3}$ For a discussion about duality in consumer theory, we refer the reader to [11].

4 A good is considered public if its use by one agent does not prevent other agents from using it [...]. (Laffont[12])
} 


\subsection{Private goods and positive externalities}

Consider an economy with $n$ consumers and $r$ private goods. The price of the good $h$ is $q_{h}$ and the consumption bundle of consumer $k$ is $x_{k}$. We write: $\ell:=r n, p:=(q, \ldots, q) \in \mathbb{R}_{++}^{\ell}$ and $x:=\left(x_{k}\right) \in \mathbb{R}_{++}^{\ell}$. Suppose that the consumption of every good for another consumer has a positive effect ${ }^{5}$ on the utility of consumer $k$. The utility function $u_{k}$ of consumer $k$ is a function of both his consumption bundle $x_{k}$ and the consumption bundles of the others $\left(x_{j}\right)_{j \neq k}$. An economic planner who wants to minimize the expenditure of the society $p \cdot x=\sum_{k=1}^{n} q \cdot x_{k}$ with respect to the individual utility levels $\left(v_{k}\right)_{k=1}^{n}$ has to solve Problem 1.1.

In Section 2, we present the assumptions used in the differentiable viewpoint in microeconomics and the definitions. In Section 3, after characterizing the solution by necessary and sufficient first order conditions, we prove the existence and continuity of the solution of Problem 1.1. In Section 4, we study the classical properties of the generalized expenditure function and we deduce that the generalized Hicksian demand is locally Lipschitz continuous. The proof relies on the result of Cornet Vial[7] on the Lipschitz behavior of the solution of a mathematical programming problem. In Section 5, we show that the generalized Hicksian demand is continuously differentiable if a strict complementarity slackness holds. Following Fiacco and McCormick[9], this result is a consequence of the Implicit Function Theorem. Finally, we obtain a Slutsky-type property for the generalized Hicksian demand.

\footnotetext{
${ }^{5}$ Recall the definition in [14]: An externality is present whenever the well-being of a consumer or the production possibilities of a firm are directly affected by the actions of another agent in the economy. Many economic goods can be considered as positive externalities such as vaccination or network.
} 


\section{Assumptions and definitions}

Let us present the assumptions on the functions $\left(u_{k}\right)_{k=1}^{n}$.

Assumption 1 For all $k=1, \ldots, n$,

1. $u_{k}$ is $C^{2}$ on $\mathbb{R}_{++}^{\ell}$,

2. $u_{k}$ is differentiably strictly quasi-concave (i.e. $D^{2} u_{k}(x)$ is negative definite on $\nabla u_{k}(x)^{\perp}$ for all $\left.x \in \mathbb{R}_{++}^{\ell}\right)$,

3. $u_{k}$ is differentiably strictly increasing (i.e. $\left.\nabla u_{k}(x) \gg 0, \forall x \in \mathbb{R}_{++}^{\ell}\right)$.

Assumption 2 For all $k \in\{1, \ldots, n\}$, if a sequence $\left(x^{\nu}\right)_{\nu \geq 0}$ converges to $x \in \partial \mathbb{R}_{++}^{\ell}$ then:

$$
\lim _{\nu \longmapsto+\infty} \frac{\nabla u_{k}\left(x^{\nu}\right) \cdot x^{\nu}}{\left\|\nabla u^{k}\left(x^{\nu}\right)\right\|}=0 .
$$

Assumption 2 on the function $u_{k}$ is weaker than the usual assumption: for all $x \in \mathbb{R}_{++}^{\ell}$, the closure in $\mathbb{R}^{\ell}$ of the set $\left\{x^{\prime} \in \mathbb{R}_{++}^{\ell} \mid u_{k}\left(x^{\prime}\right) \geq u_{k}(x)\right\}$ is contained in $\mathbb{R}_{++}^{\ell}$. The usual closure assumption implies Assumption 2 but both assumptions are not equivalent. For instance, the function $u$ defined on $\mathbb{R}_{++}^{2}$ by: $u\left(x_{1}, x_{2}\right)=\sqrt{x_{1}}+\sqrt{x_{2}}$ satisfies Assumption 2 but not the classical assumption ${ }^{7}$.

In Problem 1.1, all individual utility levels are not relevant. To determine the relevant one, we define, for $v \in \mathbb{R}^{n}$, the set $P(v)$ by:

$$
P(v):=\left\{x \in \mathbb{R}_{++}^{\ell} \mid u_{k}(x) \geq v_{k} \forall k=1, \ldots, n\right\} .
$$

If this set is empty or equal to the whole set, the vector $v$ is obviously not relevant. This motivates the definition of the set $\mathcal{V}$ by:

$$
\mathcal{V}:=\left\{v \in \mathbb{R}^{n} \mid P(v) \neq \emptyset \text { and } P(v) \neq \mathbb{R}_{++}^{\ell}\right\} .
$$

\footnotetext{
${ }^{6}$ In the paper, we use the following notations:

- $x \geq y$ means: $x_{h} \geq y_{h}$ for all $h=1, \ldots, \ell$.

- $x \gg y$ means: $x_{h}>y_{h}$ for all $h=1, \ldots, \ell$.

- $e_{h}$ denotes the h-th vector of the canonical basis of $\mathbb{R}^{\ell}$.

- $\mathbf{1}_{\ell}$ denotes the $\ell$-dimensional vector whose coordinates are all equal to one. Similarly, $\mathbf{1}_{n}$ denotes the $n$-dimensional vector whose coordinates are all equal to one. When there is no confusion, we simply write $\mathbf{1}$.

- Let $v \in \mathbb{R}^{\ell} .\|v\|:=\sum_{h=1}^{\ell}\left|v_{h}\right|$ denotes the norm of the vector $v$.

${ }^{7}$ Take $x:=\left(\frac{1}{4}, \frac{1}{4}\right)$ and $\left(x^{\nu}:=\left(1+\frac{1}{\nu}, \frac{1}{\nu}\right)\right)_{\nu \geq 1}$ as a counterexample.
} 
We give a more explicit description of $\mathcal{V}: v \in \mathcal{V}$ means: $\exists z \in \mathbb{R}_{++}^{\ell}$ such that $u_{k}(z) \geq v_{k}$ for all $k=1, \ldots, n$ and $\exists z^{\prime} \in \mathbb{R}_{++}^{\ell}, k_{0} \in\{1, \ldots, n\}$ such that: $u_{k_{0}}\left(z^{\prime}\right)<v_{k_{0}}$. Clearly, the set $\mathcal{V}$ is open ${ }^{8}$ in $\mathbb{R}^{n}$.

We conclude by a definition

Definition 1 The function e is defined on $\mathbb{R}_{++}^{\ell} \times \mathcal{V}$ by: $e(p, v)=p \cdot \Delta(p, v)$ and called the generalized expenditure function.

The next section will be devoted to the existence problem.

\section{Existence of the solution}

First, we show that the solution of Problem 1.1 is characterized by first order conditions.

\subsection{Characterization of the generalized Hicksian de- mand by first order conditions}

Proposition 1 Let $p \in \mathbb{R}_{++}^{\ell}$ and $v \in \mathcal{V}$. The two following assertions are equivalent:

1. $\bar{x}=\Delta(p, v)$

2. There exists $\lambda \in \mathbb{R}_{+}^{n} \backslash\{0\}$ such that $\bar{x}$ is the solution of the system:

$$
\left\{\begin{array}{l}
p=\sum_{k=1}^{n} \lambda_{k} \nabla u_{k}(x) \\
\lambda_{k}\left(u_{k}(x)-v_{k}\right)=0 k=1, \ldots, n \\
u_{k}(x) \geq v_{k} k=1, \ldots, n \\
x \gg 0
\end{array}\right.
$$

Proof Assertion 1 implies Assertion 2. Indeed, there exists some element $x \in \mathbb{R}_{++}^{\ell}$ such that $u_{k}(x) \geq v_{k}$ for all $k \in\{1, \ldots, n\}$ since $v$ belongs to the set $\mathcal{V}$. So by monotony of the functions $\left(u_{k}\right)_{k=1}^{n}$, there exists $\hat{x}$ such that $u_{k}(\hat{x})>v_{k}$ for all $k=1, \ldots, n$. Hence the first order conditions are necessary since Slater's constraint qualification holds ${ }^{9}$. The multiplier $\lambda:=\left(\lambda_{k}\right)_{k=1}^{n}$ is necessarily different from zero because the vector $p$ belongs to $\mathbb{R}_{++}^{\ell}$.

Now, we prove the converse statement. The functions $\left(u_{k}\right)_{k=1}^{n}$ are differentiable, quasi-concave and satisfy: $\nabla u_{k}(x) \neq 0$ for all $x \in \mathbb{R}_{++}^{\ell}$ while the objective function is linear ${ }^{10}$. This implies that the first order conditions are sufficient. Thus, Assertion 2 implies Assertion 1 .

\footnotetext{
${ }^{8}$ For the sake of completeness, this is proved in Appendix.

${ }^{9}$ See [8].

${ }^{10}$ See $[1]$.
} 


\subsection{An intermediary existence result}

To solve Problem 1.1, we study an intermediary problem. For $\varepsilon>0$ and $(p, v) \in \mathbb{R}_{++}^{\ell} \times \mathcal{V}$, we consider the following problem:

$$
\left\{\begin{array}{l}
\max -p \cdot x \\
\text { subject to } u_{k}(x) \geq v_{k}, k=1, \ldots, n \\
x_{h} \geq \varepsilon, h=1, \ldots, \ell
\end{array}\right.
$$

We start by an existence result:

Proposition 2 Let $\varepsilon>0$. The solution of Problem 3.2 denoted by $\Delta^{\varepsilon}(p, v)$ exists and is a singleton.

Proof Since $v$ belongs to $\mathcal{V}$, there exists $z_{0} \in \mathbb{R}_{++}^{\ell}$ such that $u_{k}\left(z_{0}\right) \geq v_{k}$ for all $k \in\{1, \ldots, n\}$. Let us consider $z_{1} \in \mathbb{R}^{\ell}$ such that $z_{1 h} \geq \max \left\{\varepsilon, z_{0 h}\right\}$ for all $h \in\{1, \ldots, \ell\}$. Let us consider an intermediate problem.

$$
\left\{\begin{array}{l}
\max -p \cdot x \\
\text { subject to } u_{k}(x) \geq v_{k}, k=1, \ldots, n \\
p \cdot x \leq p \cdot z_{1} \\
x \geq \varepsilon \mathbf{1} \\
x \in \mathbb{R}^{\ell}
\end{array}\right.
$$

Remark that $z_{1}$ is feasible for Problem 3.3 since $z_{1} \geq z_{0}$ and since the functions $\left(u_{k}\right)_{k=1}^{n}$ are increasing. We have the following lemma:

Lemma 1 Problem 3.2 and Problem 3.3 have the same solutions.

This result is proved in Appendix.

Now we prove that Problem 3.3 admits a solution.

The set $A:=\left\{x \in \mathbb{R}^{\ell} \mid x \geq \varepsilon \mathbf{1}\right.$ and $\left.p \cdot x \leq p \cdot z_{1}\right\}$ is a compact set as a closed and bounded set in a finite-dimensional vector space. Moreover the function $x \longmapsto-p \cdot x$ is continuous on $\mathbb{R}_{++}^{\ell}$. We conclude by Weierstrass Theorem that this problem admits a solution.

From Lemma 1, we deduce that Problem 3.2 admits a solution. To conclude, we show that the $\Delta^{\varepsilon}(p, v)$ is a singleton. Suppose that $x$ and $x^{\prime}$ are distinct solutions of Problem 3.2. The element $x^{\prime \prime}:=\frac{1}{2}\left(x+x^{\prime}\right)$ is clearly feasible. Indeed, $u_{k}\left(x^{\prime \prime}\right)>v_{k}$ for every $k=1, \ldots, n$ by strict quasi-concavity of the functions $\left(u_{k}\right)_{k=1}^{n}$ and obviously $x^{\prime \prime} \geq \varepsilon \mathbf{1}$. $x$ and $x^{\prime}$ cannot be both equal to $\varepsilon \mathbf{1}$. So, at least one of them has a component larger than $\varepsilon$. To fix the ideas, suppose that $x_{1}$ is larger than $\varepsilon$. By continuity of the functions $\left(u_{k}\right)_{k=1}^{n}$, for $\delta$ positive sufficiently small, we have: $x^{\prime \prime}-\delta e_{1} \geq \varepsilon \mathbf{1}$ and 
$u_{k}\left(x^{\prime \prime}-\delta e_{1}\right)>v_{k}$ for every $k=1, \ldots, n$. Moreover, the element $\tilde{x}:=x^{\prime \prime}-\delta e_{1}$ satisfies $-p \cdot x=-p \cdot x^{\prime \prime}<-p \cdot \tilde{x}$. So $x$ is not a solution. Consequently, one gets a contradiction.

\subsection{Continuity of $\Delta^{\varepsilon}$}

Proposition 3 Let $\varepsilon>0$. The function $\Delta^{\varepsilon}$ is continuous on $\mathbb{R}_{++}^{\ell} \times \mathcal{V}$.

Proof Let $(\bar{p}, \bar{v}) \in \mathbb{R}_{++}^{\ell} \times \mathcal{V}$ and a compact neighborhood $\Xi$ of $(\bar{p}, \bar{v})$. $\Xi$ is chosen such that $v$ belongs to $\mathcal{V}$ for all $(p, v) \in \Xi^{11}$. The compactness of $\Xi$ allows us to say that, for $M>\varepsilon$ sufficiently large, $u:=M 1$ belongs to the interior of $P(v)$ for all $(p, v) \in \Xi$. Take such a number $M$. The budget sets ${ }^{12}$ $B(p, p \cdot u)$ are all contained in a compact set $K$ since $p$ vary in a compact set contained in $\mathbb{R}_{++}^{\ell}$ when $(p, v)$ belongs to $\Xi$. For $\bar{M}>0$ large enough, $\bar{M} \mathbf{1}$ does not belong to the compact set $K$. Let us introduce the correspondence $C^{\varepsilon}$ defined, for $(p, v) \in \Xi$, by:

$C^{\varepsilon}(p, v):=\left\{x \in \mathbb{R}_{++}^{\ell} \mid u_{k}(x) \geq v_{k} \forall k=1, \ldots, n\right.$ and $\left.\varepsilon \mathbf{1} \leq x \leq \bar{M} \mathbf{1}\right\}$ and remark that, by construction, for all $(p, v) \in \Xi, \Delta^{\varepsilon}(p, v)$ is the solution of the following problem:

$$
\left\{\begin{array}{l}
\max -p \cdot x \\
\text { subject to } x \in C^{\varepsilon}(p, v)
\end{array}\right.
$$

On $\Xi$, the interior of $C^{\varepsilon}(p, v)$ is nonempty since $\mathcal{V}$ is an open set. We now prove that the function $(p, v) \longmapsto \Delta^{\varepsilon}(p, v)$ is continuous on $\Xi$. This is a consequence of Berge's Theorem[4]. We have to show that the correspondence $C^{\varepsilon}$ is both upper semi-continuous and lower semi-continuous on $\Xi$. First, we show that $C^{\varepsilon}$ is upper semi-continuous. On $\Xi$, the set $C^{\varepsilon}(p, v)$ remains in a fixed compact set. Hence the upper semi-continuity of $C^{\varepsilon}$ is equivalent to the closedness of its graph, which is a consequence of the continuity of the functions $\left(u_{k}\right)_{k=1}^{n}$.

We now have to show that the correspondence $C^{\varepsilon}$ is lower semi-continuous. Let us introduce the correspondence $\hat{C}^{\varepsilon}$ defined on $\Xi$ by: $\hat{C}^{\varepsilon}(p, v):=\{x \in$ $\mathbb{R}_{++}^{\ell} \mid u_{k}(x)>v_{k} \forall k=1, \ldots, n$ and $\left.\varepsilon \mathbf{1} \ll x \ll \bar{M} \mathbf{1}\right\}$. The correspondence $\hat{C}^{\varepsilon}$ has an open graph by the continuity of the functions $\left(u_{k}\right)_{k=1}^{n}$. So $\hat{C}^{\varepsilon}$ is lower semi-continuous. Remark that $\hat{C}^{\varepsilon}(p, v)$ is nonempty for every $(p, v) \in \Xi$ since $\bar{M} \mathbf{1}$ belongs to the interior of $P(v)$.

\footnotetext{
${ }^{11}$ This is possible when the neighborhood is small enough thanks to the openness of $\mathcal{V}$.

${ }^{12}$ Like in standard microeconomics, for $p \in \mathbb{R}_{++}^{\ell}$ and $w>0$, the budget set $B(p, w)$ is defined by: $B(p, w):=\left\{x \in \mathbb{R}_{++}^{\ell} \mid p \cdot x \leq w\right\}$.
} 
Moreover the closure of $\hat{C}^{\varepsilon}(p, v)$ is $C^{\varepsilon}(p, v)$. Indeed, let $x \in C^{\varepsilon}(p, v)$. We have to show that $x$ is the limit of a sequence of elements of $\hat{C}^{\varepsilon}(p, v)$. Let us consider $y \in \hat{C}^{\varepsilon}(p, v)$ and remark that, for all $\lambda \in(0,1),(1-\lambda) x+\lambda y$ belongs to $\hat{C}^{\varepsilon}(p, v)$ since the functions $\left(u_{k}\right)_{k=1}^{n}$ are strictly quasi-concave. To conclude, $x$ is the limit of the sequence $\left(x^{\nu}:=\left(1-\frac{1}{\nu}\right) x+\frac{1}{\nu} y\right)_{\nu \geq 1}$. Moreover, one remarks that $x^{\nu}$ belongs to $\hat{C}^{\varepsilon}(p, v)$ for all $\nu \geq 1$, hence the result.

We deduce that the correspondence $C^{\varepsilon}$ is lower semi-continuous since the closure of a lower semi-continuous correspondence is lower semi-continuous ${ }^{13}$. Berge's Theorem implies that the function $\Delta^{\varepsilon}$ is continuous on the set $\Xi$, hence on $\mathbb{R}_{++}^{\ell} \times \mathcal{V}$. $\square$

\subsection{Characterization of $\Delta^{\varepsilon}(p, v)$ by first order condi- tions}

Let $\varepsilon>0$. The first order conditions associated to Problem 3.2 are: There exists $\lambda^{\varepsilon} \in \mathbb{R}_{+}^{n}$ and $\mu^{\varepsilon} \in \mathbb{R}_{+}^{\ell}$ such that $\Delta^{\varepsilon}(p, v)$ is the solution of the system:

$$
\left\{\begin{array}{l}
p=\sum_{k=1}^{n} \lambda_{k}^{\varepsilon} \nabla u_{k}(x)+\mu^{\varepsilon} \\
\lambda_{k}^{\varepsilon}\left(u_{k}(x)-v_{k}\right)=0, k=1, \ldots, n \\
u_{k}(x) \geq v_{k}, k=1, \ldots, n \\
\mu_{h}^{\varepsilon}\left(\varepsilon-x_{h}\right)=0, h=1, \ldots, \ell \\
x_{h} \geq \varepsilon, h=1, \ldots, \ell
\end{array}\right.
$$

As before, the first order conditions are necessary since Slater's constraint qualification holds. These are sufficient since the objective function is linear, the functions $\left(u_{k}\right)_{k=1}^{n}$ are quasi-concave functions satisfying $\nabla u_{k}(x) \neq 0$ for all $x \in \mathbb{R}_{++}^{\ell}$ and the $\ell$ additional constraints are affine.

\subsection{Existence and continuity of the solution of Prob- lem 1.1}

In this subsection, we show the main result of the section:

Proposition 4 For $(p, v) \in \mathbb{R}_{++}^{\ell} \times \mathcal{V}, \Delta(p, v)$ is a singleton. Moreover, the function $\Delta$ is continuous on $\mathbb{R}_{++}^{\ell} \times \mathcal{V}$.

\footnotetext{
${ }^{13}$ See the appendix of [10].
} 
Proof Let $(p, v) \in \mathbb{R}_{++}^{\ell} \times \mathcal{V}$ and a compact neighborhood $\Xi$ of $(p, v)$. As before, the set $\Xi$ is chosen such that $v^{\prime}$ belongs to $\mathcal{V}$ for all $\left(p^{\prime}, v^{\prime}\right) \in \Xi$. Our goal is to show that there exists $\bar{\varepsilon}>0$ such that the multipliers $\mu^{\bar{\varepsilon}}$ associated to the additional constraints are equal to zero for all $\left(p^{\prime}, v^{\prime}\right)$ in $\Xi$. We reason by contradiction. Otherwise, there would exist a decreasing sequence $\left(\varepsilon_{q}\right)_{q \geq 0}$ that converges to zero and a sequence of $\Xi$ denoted by $\left(p_{q}, v_{q}\right)_{q \geq 0}$ such that $\mu^{\varepsilon_{q}}:=\mu^{\varepsilon_{q}}\left(p_{q}, v_{q}\right) \neq 0$ for all $q \in \mathbb{N}$.

Necessarily, $\left(x_{q}:=\Delta^{\varepsilon_{q}}\left(p_{q}, v_{q}\right)\right)_{q \geq 0}$ is bounded. Indeed, for all $q \in \mathbb{N}$, $x_{q} \gg 0$ and $a \cdot x_{q} \leq p_{q} \cdot x_{q} \leq p_{0} \cdot x_{0}$ where the vector $a$ is defined by $a_{h}:=\min \left\{p_{h}^{\prime} \mid\left(p^{\prime}, v^{\prime}\right) \in \Xi\right\}$ for $h=1, \ldots, \ell$. The vector $a$ is well defined and belongs to $\mathbb{R}_{++}^{\ell}$ thanks to the compactness of $\Xi$. Therefore the sequence $\left(x_{q}\right)_{q \geq 0}$ converges, up to a subsequence, to an element $\hat{x}$ belonging to the boundary of $\mathbb{R}_{++}^{\ell}$ and the sequence $\left(p_{q}, v_{q}\right)_{q \geq 0}$ converges, up to a subsequence, to some element $(\hat{p}, \hat{v}) \in \Xi$ since $\Xi$ is a compact set. In particular, remark that $\hat{v}$ belongs to $\mathcal{V}$ and that $\hat{p}$ is different from zero. With a slight abuse of notation, we denote the converging subsequences as the original sequences.

Remark that the sequence $\left(\mu^{\varepsilon_{q}}\right)_{q \geq 0}$ is also bounded thanks to the first equation of 3.5 and to the compactness of $\Xi$. From the same equation, recalling that we consider the 1 -norm, we have ${ }^{14}$, for all $k \in\{1, \ldots, n\}$ and all $q \in \mathbb{N}$ :

$$
\lambda_{k}^{\varepsilon_{q}}\left\|\nabla u_{k}\left(x_{q}\right)\right\| \leq\left\|p_{q}\right\|
$$

Therefore, for all $k \in\{1, \ldots, n\}$ and all $q \in \mathbb{N}$, we have:

$$
0 \leq \lambda_{k}^{\varepsilon_{q}} \frac{\nabla u_{k}\left(x_{q}\right) \cdot x_{q}}{\left\|p_{q}\right\|} \leq \frac{\nabla u_{k}\left(x_{q}\right) \cdot x_{q}}{\left\|\nabla u_{k}\left(x_{q}\right)\right\|} .
$$

Thanks to Assumption 2 and to the previous inequalities, for all $k \in\{1, \ldots, n\}$, we have:

$$
\lim _{q \longmapsto+\infty} \lambda_{k}^{\varepsilon_{q}} \frac{\nabla u_{k}\left(x_{q}\right) \cdot x_{q}}{\left\|p_{q}\right\|}=0 .
$$

For $q \in \mathbb{N}$, doing an inner product with $x_{q}$ and dividing by $\left\|p_{q}\right\|$ in the first equation of 3.5 , one obtains:

$$
\frac{p_{q} \cdot x_{q}}{\left\|p_{q}\right\|}=\sum_{k=1}^{n} \lambda_{k}^{\varepsilon_{q}} \frac{\nabla u_{k}\left(x_{q}\right) \cdot x_{q}}{\left\|p_{q}\right\|}+\frac{1}{\left\|p_{q}\right\|} \mu^{\varepsilon_{q}} \cdot x_{q} .
$$

Moreover, from 3.5, $\mu^{\varepsilon_{q}} \cdot x_{q}=\varepsilon_{q} \mu^{\varepsilon_{q}} \cdot \mathbf{1}=\varepsilon_{q}\left\|\mu^{\varepsilon_{q}}\right\|$ converges to zero. So the right-hand side goes to zero. Therefore the left-hand side goes to zero. Since

\footnotetext{
${ }^{14}$ We write $\lambda_{k}^{\varepsilon_{q}}:=\lambda_{k}^{\varepsilon_{q}}\left(p_{q}, v_{q}\right)$ for $k \in\{1, \ldots, n\}$ and $q \in \mathbb{N}$ to simplify the notation.
} 
$(\hat{p}, \hat{v})$ belongs to $\Xi, \hat{p} \gg 0$ and the limit of the sequence $\left(x_{q}\right)_{q \geq 0}$ is necessarily zero.

Let $\bar{x} \in \mathbb{R}_{++}^{\ell}$. We show that $\bar{x}$ belongs to $P(\hat{v})$. For $q$ sufficiently large, one has: $\bar{x} \gg x_{q}$ and $\bar{x} \gg \varepsilon_{q} 1$. Thus by monotony of the functions $\left(u_{k}\right)_{k=1}^{n}$, for all $k \in\{1, \ldots, n\}, u_{k}(\bar{x})>u_{k}\left(x_{q}\right) \geq v_{k q}$. Thus $\bar{x}$ belongs to $P\left(v_{q}\right)$ for $q$ large enough. By continuity of the functions $\left(u_{k}\right)_{k=1}^{n}, \bar{x}$ belongs to $P(\hat{v})$. Since $\bar{x}$ was arbitrarily chosen, we have: $P(\hat{v})=\mathbb{R}_{++}^{\ell}$ which contradicts $\hat{v} \in \mathcal{V}$.

Therefore, there exists $\bar{\varepsilon}>0$ such that $\mu^{\bar{\varepsilon}}=0$. Thus, $\Delta^{\bar{\varepsilon}}\left(p^{\prime}, v^{\prime}\right)$ satisfies the necessary and sufficient conditions associated to 4.1 for all $\left(p^{\prime}, v^{\prime}\right)$ in $\Xi$. So $\Delta=\Delta^{\bar{\varepsilon}}$ on $\Xi$ and the continuity of $\Delta$ follows.

\section{Properties of $\Delta$ and $e$.}

\subsection{Properties of $e$}

Now, we study the properties of the function $e$.

Proposition 5 [Properties of e]

1. The function e is concave in $p$.

2. The function e is twice differentiable a.e. and $D^{2} e(p, v)$ is semi-definite negative when defined.

3. $D_{p} e(p, v)=\Delta(p, v)$ and $D_{p}^{2} e(p, v)=D_{p} \Delta(p, v)$ when defined.

Proof The proof is essentially borrowed from Rader[15]. The function $-e$ is convex in $p$ as a maximum of linear functions. Indeed for $(p, v) \in \mathbb{R}_{++}^{\ell} \times \mathcal{V}$, $e(p, v)$ can be defined by $-e(p, v)=\max \left\{-p \cdot y \mid y \in \mathbb{R}_{++}^{\ell}, u_{k}(y) \geq v_{k}, k=\right.$ $1, \ldots, n\}$. So the function $e$ is concave. By Alexandroff's Theorem, the function $e$ is twice differentiable a.e. in $p$ and its second derivative is semidefinite negative.

By Theorem 4(iii) of Rader[15], $D_{p} e(p, v)=\Delta(p, v)$

\subsection{Lipschitz behavior of $\Delta$}

Firstly, for all $(p, v) \in \mathbb{R}_{++}^{\ell} \times \mathcal{V}$, we define the set $M(p, v)$ by:

$$
M(p, v):=\left\{k \in\{1, \ldots, n\} \mid u_{k}(x)=v_{k}\right\} .
$$


Secondly, let us define the set $\Pi$ by:

$$
\Pi:=\left\{(p, v) \in \mathbb{R}_{++}^{\ell} \times \mathcal{V} \mid\left(\nabla u_{k}(\Delta(p, v))\right)_{k \in M(p, v)} \text { are independent. }\right\} .
$$

This set is an open subset of $\mathbb{R}^{\ell} \times \mathbb{R}^{n}$ thanks to the continuity of $\Delta$ on the open subset $\mathbb{R}_{++}^{\ell} \times \mathcal{V}$.

For $(p, v) \in \Pi$, the constraints of the optimization problem satisfy the Linear Independence constraint qualification(LICQ $)^{15}$. Thus, the multipliers are unique and the following definition makes sense. For $(p, v) \in \Pi$, we define the set $K(p, v)$ by:

$$
K(p, v):=\left\{k \in\{1, \ldots, n\} \mid \lambda_{k}(p, v)>0\right\} .
$$

From Proposition 1, one deduces that this set is nonempty. The cardinal of $K(p, v)$ is denoted by $\kappa(p, v)$.

Finally, the set $\tilde{\Pi}$ is defined by:

$$
\tilde{\Pi}:=\{(p, v) \in \Pi \mid K(p, v)=M(p, v)\} .
$$

Proposition 6 The function $\Delta$ and the multipliers $\left(\lambda_{k}\right)_{k=1}^{n}$ are locally Lipschitz continuous on $\Pi$. Hence, the function $\Delta$ is differentiable almost everywhere on $\Pi$.

Proof This is an application of Cornet and Vial's result[7]. To show that the function $\Delta$ is locally Lipschitz on $\Pi$, we check that Assumptions (A.0), (C.1) and (C.2) of Corollary 2.3. of [7] are satisfied. We define on $\mathbb{R}_{++}^{\ell} \times \Pi$ the following functions:

- $f(x, p, v):=p \cdot x$,

- $g_{k}(x, p, v):=v_{k}-u_{k}(x)$ for $k \in\{1, \ldots, n\}$.

For $(p, v) \in \Pi, \Delta(p, v)$ is the solution of the problem:

$$
\left\{\begin{array}{l}
\min f(x, p, v) \\
\text { subject to } \\
g_{k}(x, p, v) \leq 0, k=1, \ldots, n
\end{array}\right.
$$

$x$ is the variable and $(p, v)$ are the parameters.

Assumptions (A.0) are satisfied. Indeed, we take $U=\mathbb{R}_{++}^{\ell}$ and $P=\Pi$. The set $U$ is an open set and the set $P$ is obviously a metric space. So Assumption (A.0) (i) is satisfied. Assumptions (A.0) (ii), (iii), (iv) and (v) are

\footnotetext{
${ }^{15}$ See [8].
} 
satisfied because the functions at stake are $C^{2}$ on the set $U \times P$. Assumption (A.0) (vi) is satisfied with $Q=C=-\mathbb{R}_{+}^{n}$.

Assumption (C.1) is satisfied. This is an immediate consequence of the definition of $\Pi$.

Assumption (C.2) is satisfied. Let $x$ be a solution of Problem 4.1 with an associated multiplier $\lambda:=\left(\lambda_{k}\right)_{k=1}^{n}$. We have to check that, for all $h \in \mathbb{R}^{\ell}$, $h \neq 0$ such that: $\nabla f(x, p, v) \cdot h=0$ and $\nabla g_{k}(x, p, v) \cdot h=0$ for $k \in K(p, v)$, we have ${ }^{16}$ :

$$
\left.\left[D^{2} f(x, p, v)+\sum_{k \in K(p, v)} \lambda_{k} D^{2} g_{k}, x, p, v\right)\right] h \cdot h>0 .
$$

Since we have : $D^{2} f \equiv 0$, it remains to show that:

$$
\sum_{k \in K(p, v)} \lambda_{k} D^{2} g_{k}(x, p, v) h \cdot h>0 .
$$

that is:

$$
-\sum_{k \in K(p, v)} \lambda_{k} D^{2} u^{k}(x) h \cdot h>0
$$

which is true because of Assumption 1 and because $\nabla u^{k}(x) \cdot h=0$ for $k \in K(p, v)^{17}$.

From [7], the function $\Delta$ is locally Lipschitz on $\Pi$ and by Rademacher's Theorem, the function $\Delta$ is almost everywhere differentiable on $\Pi$. $\square$

\section{Differential properties of $\Delta$}

\subsection{Continuous differentiability of $\Delta$}

Proposition 7 If $(\bar{p}, \bar{v}) \in \tilde{\Pi}, \Delta$ is continuously differentiable on a neighborhood of $(\bar{p}, \bar{v})$.

Proof This proof is essentially an application of the Implicit Function Theorem. It is quite standard borrowing ideas from Fiacco-McCormick[9]. Without loss of generality, suppose that: $M(\bar{p}, \bar{v})=\{1, \ldots, r\}$. Remark that, by continuity of $\Delta$ and the utility functions $\left(u_{k}\right)_{k=1}^{n}$, we can neglect the non satiated constraints and, by continuity of the positive multipliers, one

\footnotetext{
${ }^{16}$ Thanks to Proposition $1, K(p, v)$ is nonempty.

${ }^{17}$ Indeed, $K(p, v)$ is a subset of $M(p, v)$
} 
has: $M(p, v)=M(\bar{p}, \bar{v})$ on a neighborhood of $(\bar{p}, \bar{v})$. As shown above, since the first order optimality conditions are necessary and sufficient and since the functions $\left(u_{k}\right)_{k=1}^{n}$ are continuous, the element $\Delta(p, v)$ and the associated multiplier $\lambda(p, v)$ are solution of the equation $G(x, \lambda, p, v)=0$ where $G$ is defined by:

$$
G(x, \lambda, p, v)=\left\{\begin{array}{l}
p-\sum_{k=1}^{r} \lambda_{k} \nabla u_{k}(x) \\
u_{k}(x)-v_{k} k=1, \ldots, r
\end{array}\right.
$$

To show that the function $\Delta$ and the multiplier are continuously differentiable on a neighborhood of $(\bar{p}, \bar{v})$, from the Implicit Function Theorem, it suffices to show that the partial Jacobian matrix of $G$ with respect to $(x, \lambda)$ has full column $\operatorname{rank}^{18}$ at $\bar{x}:=\Delta(\bar{p}, \bar{v})$. This matrix is equal to:

$$
M:=\left[\begin{array}{ccccc}
-\sum_{k=1}^{n} \bar{\lambda}_{k} D^{2} u^{k}(\bar{x}) & -\nabla u_{1}(\bar{x}) & \ldots & \ldots & -\nabla u_{r}(\bar{x}) \\
\nabla u_{1}(\bar{x})^{T} & 0 & \ldots & \ldots & 0 \\
\vdots & \vdots & \vdots & \vdots & \vdots \\
\nabla u_{r}(\bar{x})^{T} & 0 & \ldots & \ldots & 0
\end{array}\right]
$$

It is sufficient to prove that $M\left(\begin{array}{c}\Delta x \\ \Delta \lambda\end{array}\right)=0$ implies: $\Delta x=0$ and $\Delta \lambda=0 . \Delta x$ is a column vector of dimension $\ell$ and $\Delta \lambda$ is a column vector of dimension $r$. We have to solve the system:

$$
\left\{\begin{array}{l}
-\sum_{k=1}^{r} \bar{\lambda}_{k} D^{2} u_{k}(\bar{x}) \Delta x-\sum_{k=1}^{r} \Delta \lambda_{k} \nabla u_{k}(\bar{x})=0 \\
\nabla u_{k}(\bar{x}) \cdot \Delta x=0, \forall k=1, \ldots, r
\end{array}\right.
$$

We multiply the first line by $(\Delta x)^{T}$ :

$$
\left\{\begin{array}{l}
-\sum_{k=1}^{r} \bar{\lambda}_{k}(\Delta x)^{T} D^{2} u_{k}(\bar{x}) \Delta x-\sum_{k=1}^{r} \Delta \lambda_{k} \nabla u_{k}(\bar{x}) \cdot \Delta x=0 \\
\nabla u_{k}(\bar{x}) \cdot \Delta x=0, \forall k=1, \ldots, r
\end{array}\right.
$$

Thus we have:

$$
\left\{\begin{array}{c}
-\sum_{k=1}^{r} \bar{\lambda}_{k}(\Delta x)^{T} D^{2} u_{k}(\bar{x}) \Delta x=0 \\
\nabla u_{k}(\bar{x}) \cdot \Delta x=0, \forall k \in\{1, \ldots, r\}
\end{array}\right.
$$

\footnotetext{
${ }^{18}$ The vectors are, by convention, column vectors and the transpose of a vector $x$ is denoted by $x^{T}$. We use the notation: $\bar{\lambda}:=\lambda(\bar{p}, \bar{v})$.
} 
For all $k \in\{1, \ldots, r\}, D^{2} u_{k}(\bar{x})$ is negative definite on $\nabla u_{k}(\bar{x})^{\perp}$ and since $\Delta x \in \nabla u_{k}(\bar{x})^{\perp}$, we have : $\Delta x=0$. Hence, the first equation becomes:

$$
-\sum_{k=1}^{r} \Delta \lambda_{k} \nabla u_{k}(\bar{x})=0
$$

and we conclude that $\Delta \lambda=0$ since $(\bar{p}, \bar{v}) \in \Pi$.

\subsection{Slutsky-type property}

The next result is a generalization of the well known result about the negative definiteness of the Slutsky matrix.

Proposition 8 Suppose that $(\bar{p}, \bar{v}) \in \tilde{\Pi}$. The matrix $D_{p} \Delta(\bar{p}, \bar{v})$ has rank $\ell-\kappa(\bar{p}, \bar{v})$ and its kernel is the linear space $\mathcal{L}\left(\nabla u^{k}(\bar{x}), k \in M(\bar{p}, \bar{v})\right)$ spanned by the family $\left(\nabla u_{k}(\bar{x})\right)_{k \in M(\bar{p}, \bar{v})}$ where $\bar{x}:=\Delta(\bar{p}, \bar{v})$.

Proof Thanks to Proposition $5, D_{p} \Delta(\bar{p}, \bar{v})$ defines a symmetric negative semi-definite bilinear form. Remark that, by continuity of $\Delta$, we can neglect the non satiated constraints and, by continuity of the positive multipliers, $M(p, v)=M(\bar{p}, \bar{v})$ on a neighborhood of $(\bar{p}, \bar{v})$.

Without loss of generality, suppose that $M(\bar{p}, \bar{v})=\{1, \ldots, r\}$. For $p \in$ $\mathbb{R}_{++}^{\ell}$ sufficiently near from $\bar{p}, \Delta(p, \bar{v})$ is characterized by the first order conditions ${ }^{19}$ :

- $u_{k}(\Delta(p, \bar{v}))=\bar{v}^{k}, \forall k \in\{1, \ldots, r\}$,

- $p=\sum_{k=1}^{n} \alpha_{k}(p) \nabla u_{k}\left(\Delta_{K}(p, \bar{v})\right)$ with $\alpha_{k}(p)>0 \forall k=1, \ldots, r$.

We differentiate the first condition with respect to $p$ and obtain at $\bar{p}$ for all $q \in \mathbb{R}^{\ell}$ :

$$
\nabla u_{k}(\Delta(\bar{p}, \bar{v})) \cdot D_{p} \Delta(\bar{p}, \bar{v})(q)=\nabla u_{k}(\bar{x}) \cdot D_{p} \Delta(\bar{p}, \bar{v})(q)=0 \forall k=1, \ldots, r .
$$

These equalities tell us that the image of $D_{p} \Delta(\bar{p}, \bar{v})$ is contained in the linear subspace $\cap_{k=1}^{r} \nabla u_{k}(\bar{x})^{\perp}$ of dimension $\ell-r$ since $(\bar{p}, \bar{v})$ belongs to $\Pi$. Furthermore, since $D_{p} \Delta(\bar{p}, \bar{v})$ defines a symmetric negative semi-definite bilinear form, $\nabla u_{k}(\bar{x})$ belongs to the kernel of $D_{p} \Delta(\bar{p}, \bar{v})$ for all $k=1, \ldots, r$. Thus, the dimension of the image of $D_{p} \Delta(\bar{p}, \bar{v})$ is at most $\ell-r$. We differentiate the second condition with respect to $p$. We have for $q \in \mathbb{R}^{\ell}$ :

$$
q=\sum_{k=1}^{r} \alpha_{k}(p) D^{2} u_{k}(\Delta(p, \bar{v})) D_{p} \Delta(p, \bar{v})(q)+\sum_{k=1}^{n}\left(\nabla \alpha_{k}(p) \cdot q\right) \nabla u_{k}(\Delta(p, \bar{v})) .
$$

\footnotetext{
${ }^{19}$ To simplify the notation, we write $\alpha_{k}(p):=\lambda(p, \bar{v})$ for all $k=1, \ldots, r$.
} 
For all $q \in \cap_{k=1}^{r} \nabla u_{k}(\bar{x})^{\perp}$, we have:

$$
q=\left[\sum_{k=1}^{r} \alpha_{k}(\bar{p}) D^{2} u_{k}(\bar{x})\right] D_{p} \Delta(\bar{p}, \bar{v})(q) .
$$

So, we have for $q \in \cap_{k=1}^{r} \nabla u_{k}(\bar{x})^{\perp}$ :

$$
D_{p} \Delta(\bar{p}, \bar{v})(q)=0 \Longrightarrow q=0 .
$$

So the kernel of the restriction on $\cap_{k=1}^{r} \nabla u_{k}(\bar{x})^{\perp}$ of $D_{p} \Delta(\bar{p}, \bar{v})$ is reduced to zero. So, the rank of $D_{p} \Delta(\bar{p}, \bar{v})$ is at least $\ell-r$. Hence, the rank of $D_{p} \Delta(\bar{p}, \bar{v})$ is equal to $\ell-r$ and the kernel of $D_{p} \Delta(\bar{p}, \bar{v})$ is equal to $\mathcal{L}\left(\nabla u_{k}(\bar{x}), k=1, \ldots, r\right)$.

\section{Conclusion}

This paper provides a new study of the classical expenditure minimization problem. First remark that, in the classical case $n=1$, obviously we have: $\Pi=\mathbb{R}_{++}^{\ell} \times \mathcal{V}$. It is very important to note that the main requirement for the Lipschitz behavior is the linear independence of the gradients while the continuous differentiability requires moreover a strict complementarity slackness condition. In view of the applications, we propose a framework for which we have: $\Pi=\mathbb{R}_{++}^{\ell} \times \mathcal{V}$. Suppose that, for $k:=1, \ldots, n$,

$$
u_{k}(x):=\sum_{h=1}^{\ell} a_{k h} b_{h}\left(x_{h}\right)
$$

where the functions $\left(b_{h}\right)_{h=1}^{\ell}$ are twice continuously real-valued functions such that: $b_{h}^{\prime}>0$ and $b_{h}^{\prime \prime}<0$ and $\sum_{h=1}^{\ell} a_{h}=1$ for all $k \in\{1, \ldots, n\}$. As it was shown in [6], the only requirement is that $A:=\left(a_{k h}\right)$ has full row rank. It can be related to decision theory through the expected utility (if $b_{h}:=b$ for all $h \in\{1, \ldots, \ell\})$ or to the separable preferences in microeconomics. We refer to [14] for a discussion about the expected utility and to [3] for a presentation of separable preferences. An open question is to find how "big" is the set $\tilde{\Pi}$ in the set $\Pi$. In particular, one would know under which conditions the set $\tilde{\Pi}$ has full Lebesgue measure in $\Pi$.

\section{Appendix}

\section{Openness of $\mathcal{V}$}

Lemma 2 The set $\mathcal{V}$ is an open set. 
Proof Let $v^{0} \in \mathcal{V}$. We want to construct a neighborhood of $v^{0}$ contained in $\mathcal{V}$. There exists $x^{0} \in \mathbb{R}_{++}^{\ell}$ such that: $u_{k}\left(x^{0}\right) \geq v_{k}^{0}$ for all $k=1, \ldots, n$ and there exists $y^{0} \in \mathbb{R}_{++}^{\ell}$ and $k_{0} \in\{1, \ldots, n\}$ such that: $u_{k_{0}}\left(y^{0}\right)<v_{k_{0}}^{0}$. Let us define $\underline{v}$ by $\underline{v}:=v^{0}-\mathbf{1}_{n}$ for $k=1, \ldots, n$ and $\bar{v}$ by $\bar{v}_{k}:=u_{k}\left(x^{0}+\mathbf{1}_{\ell}\right)$ for $k=1, \ldots, n$. Finally, we define the sets $A:=\left\{v \in \mathbb{R}^{n} \mid u_{k_{0}}\left(y^{0}\right)<v_{k_{0}}\right\}$ and $\left.B:=\prod_{k=1}^{n}\right] \underline{v}_{k}, \bar{v}_{k}[$. By construction the set $A \cap B$ is a nonempty open neighborhood of $v^{0}$ contained in $\mathcal{V}$. Since $v^{0}$ was arbitrarily chosen, one concludes that the set $\mathcal{V}$ is an open set.

\section{Proof of Lemma 1}

Let us justify that Problem 3.2 and Problem 3.3 have the same solutions. Let $y$ be a solution of Problem 3.2. Since $z_{1}$ is feasible for Problem 3.2, one has: $p \cdot y \leq p \cdot z_{1}$. So $y$ is feasible for Problem 3.3 and obviously $y$ is a solution to Problem 3.3. Indeed, the set of feasible points of Problem 3.3 is contained in the set of feasible points of Problem 3.2.

Let $y$ be a solution of Problem 3.3, $y$ is feasible for Problem 3.2 by construction. Let $z$ be feasible for Problem 3.2, either $-p \cdot z<-p \cdot z_{1}$ or $-p \cdot z \geq-p \cdot z_{1}$. In the first case, obviously, $-p \cdot z \leq-p \cdot y$. In the second case, $z$ is feasible for Problem 3.3. Thus, $-p \cdot z \leq-p \cdot z_{1} \leq-p \cdot y$. To conclude, $y$ is a solution to Problem 3.2.

\section{References}

[1] Arrow, K.J., Enthoven, A. C.: Quasi-concave Programming. Econometrica 29(4), 779-800 (1961)

[2] Aumann, R.: Utility theory without the completeness assumption. Econometrica 30, 445-462 (1962)

[3] Barten, A., Böhm, V.: Consumer Theory. Handbook of mathematical economics, Vol. 2,pp 381-429, Ch. 9. Amsterdam: North-Holland 1982

[4] Berge, C.: Topological spaces. New York: Dover publications Mineola 1997

[5] Bewley, T.: Knightian Decision Theory: Part I. Decisions in Economics and Finance, 25, (2002), 79-110 
[6] Biheng, N., Bonnisseau, J.-M.: Regular economies with ambiguity aversion. Documents de Travail du Centre d'Economie de la Sorbonne 2013.83

[7] Cornet, B., Vial, J.P.: Lipschitzian solutions of perturbed non linear problems. SIAM Journal of control and optimization 24 (6) 1986

[8] Eustaquio, R.G., Karas, E. W., Ribeiro, A. A.: Constraint qualifications for nonlinear programming. Technical report, Federal University of Parana, 2010

[9] Fiacco, A., McCormick, G.: Nonlinear programming: Sequential unconstrained minimization techniques. Classics in Applied Mathematics, Vol. 4. Philadelphia: SIAM 1990

[10] Florenzano, M.: General equilibrium analysis: Existence and optimality properties of equilibria. Kluwer Academic Publishers 2003

[11] Diewert, E.W.: Duality approaches to microeconomic theory. Handbook of mathematical economics, Vol. 2, Ch. 12. Amsterdam: North-Holland 1982

[12] Laffont, J.-J: Fundamentals of Public Economics. Cambridge: The MIT Press Cambridge 1988

[13] Mas-Collel, A.: The Theory of General Economic Equilibrium: A Differentiable Approach. Cambridge: Cambridge University Press 1985

[14] Mas-Colell, A., Whinston, M.D., Green, J.R.: Microeconomic Theory. New York Oxford: Oxford University Press 1995

[15] Rader, R.: Nice demand functions. Econometrica 41(5), 913-935 (1973) 